\title{
Presencia de la información audiovisual en los periódicos digitales españoles
}

\author{
Gloria DURÁN PILA \\ gduranpi@alumnos.unex.es \\ Universidad de Extremadura. Alumna de Máster Universitario de Investigación en Ciencias \\ Sociales y Jurídicas \\ Jorge CALDERA SERRANO \\ jcalser@alcazaba.unex.es \\ Universidad de Extremadura. Departamento de Información y Comunicación
}

Recibido: $17 / 04 / 2014$

Aceptado: 25/05/2014

\section{RESUMEN}

Se realiza un estudio sobre los principales periódicos españoles presentes en la red con el fin de determinar la utilización de información audiovisual en estos medios, determinando así la clara hibridación entre los diferentes formatos de los medios de información tradicional; igualmente se analiza la fuente de procedencia de dichas piezas audiovisuales para determinar si dicha información es de elaboración propia, ha sido adquirida a otros medios de comunicación o agencias informativas u obtenidas directamente de la red. La hipótesis inicial queda validada al observar la presencia de este tipo de formato en los medios, siendo la procedencia tanto interna como externa.

Palabras clave: Información audiovisual, Periodismo digital, hibridación de medios, Fuentes audiovisuales de información, Periódicos españoles en línea

\section{Presence of audiovisual information in Spanish digital newspaper}

\begin{abstract}
It is done a study about the major Spanish newspapers on the network in order to define the use of audiovisual information in these media, determining with that the clear hybridization between different formats of traditional media; in the same way it is analyzed the source of these audiovisual pieces to determine if this information is made by itself, it has been acquired from the other media or news agency or it has been obtained directly from the network. The initial hypothesis is validated when observing the presence of this kind of format in the media, being the origin as internal as external.
\end{abstract}

Keywords: Audiovisual Information, Digital journalism, media hybridization, audiovisual sources, Spanish newspapers online

\section{LOS MEDIOS IMPRESOS DE COMUNICACIÓN Y SU EVOLUCIÓN POR MEDIO DE LA RED}

En las últimas décadas del siglo XXI se ha producido una gran evolución en los canales de información y de comunicación impulsada, sobre todo, por la 
herramienta Internet. La Red de redes ha globalizado y cambiado nuestra manera de conocer y de entender el mundo que nos rodea, dando paso a una nueva realidad social en la que se ha afianzado el modelo de la Sociedad de la Información, existente antes de la aparición del ciberespacio.

El desarrollo de este nuevo panorama social no sería posible sin el avance de las tecnologías de la información y la comunicación. Éstas han incorporado e incorporan a nuestras vidas nuevos conceptos y nuevos mecanismos informacionales y comunicativos, los cuales son asimilados de manera innata por los "nativos digitales" (Prensky, 2001) mientras que las generaciones anteriores deben realizar notables esfuerzos en un proceso continuo de asimilación.

Todas estas nuevas herramientas globales facilitan el acceso a la información y su utilización en cualquier parte del planeta, potenciando los aspectos audiovisuales y multimedia, generando un canal bidireccional de comunicación explotado en las webs 2.0 y en las redes sociales.

Esta nueva realidad global no ha pasado desapercibida en el panorama empresarial, que se ha visto abocado a incluir nuevas formas de producción. Un cambio productivo que incluye también al sector de los medios de comunicación y, por tanto, a la difusión de la información (Salaverría \& García, 2008). Como consecuencia, aparece un nuevo concepto mediático, surgido de las nuevas necesidades, con cada vez mayor potencialidad: el Periodismo Digital (Álvarez, 2003). Este modelo periodístico, diferente al tradicional, posibilita nuevas formas de tratar y de ofrecer la información atendiendo a las nuevas características de su audiencia.

El Periodismo Digital (Martínez, 2001) es un concepto implantado en la Sociedad de la Información, pero, como el resto de mecanismos y herramientas comunicativos, continúa en proceso de desarrollo. Ya hay medios que han rechazado publicar por las vías tradicionales, teniendo como única fórmula de venta o difusión la presencia en la Red.

\section{JUSTIFICACIÓN DEL TRABAJO}

La explosión de Internet y las nuevas necesidades de la Sociedad de la Información han hecho que, al final, todos los medios de comunicación tradicionales, como son la radio, la televisión y los periódicos, tengan su espacio en la Red. Estos en su edición online han tenido que adaptar sus contenidos a los recursos del ciberespacio, dando lugar a una hibridación de medios en la que convergen vídeos, audios, fotografías y textos. Paralelo a este proceso de adaptación, surgieron nuevos medios digitales creados únicamente para ser desarrollados en la web, con total libertad a la hora de elaborar contenidos en diferentes formatos.

Uno de los múltiples recursos de los que disponen los medios digitales son los elementos audiovisuales, los cuales facilitan la comprensión de la información al 
usuario, en el caso de las noticias, y ofrecen contenidos diferentes a los informativos que sirven como reclamo del medio.

El empleo de los vídeos en los periódicos digitales que han proliferado en los últimos años hace necesario realizar un estudio para determinar cuál es su presencia en los diarios digitales, así como su finalidad, la validez de sus contenidos y por tanto, de sus fuentes. Además, también hace falta abordar en él otro aspecto como es el del espacio que dedican estos medios a sus archivos audiovisuales.

\section{OBJETIVOS Y METODOLOGÍA}

En este trabajo queremos abordar una serie de objetivos que serán analizados en el trabajo de campo posterior:

Objetivo 1: Determinar la presencia de material audiovisual en los periódicos digitales:

Lo primero que hay que corroborar en este estudio es el empleo de los recursos audiovisuales por parte de los diarios digitales.

Objetivo 2: Determinar el uso de las fuentes internas y externas de los vídeos de los diarios digitales:

Comprobado el empleo de información audiovisual en los periódicos digitales, habrá que determinar en qué medida esta viene dada por fuentes propias o por fuentes externas al medio.

Objetivo 3: Determinar la procedencia de las fuentes externas audiovisuales de los periódicos digitales analizados:

Averiguar de dónde procede la información audiovisual que no ha sido creada por el propio periódico digital sino adquirida de empresas o agencias externas. Esto marcará la diferencia, o no, entre unos diarios online y otros.

La metodología del trabajo se compone de una revisión bibliográfica y un trabajo de campo realizado de forma secuencial. Ambas partes han sido estructuradas por medio de las siguientes fases:

Fase 1. Revisión bibliográfica y análisis de fuentes

Para la búsqueda de información se han consultado las bases de datos más relevantes y los catálogos de las bibliotecas universitarias más cercanos. Hemos encontrado dos artículos muy útiles que nos han servido de referencia del año 2012 de Matilde Roa Amaya y de Jorge Caldera Serrano, en los que analizan los medios digitales que publican también en papel, asi como un trabajo de fin de máster relacionado con la materia elaborado también por Matilde Roa Amaya (2011) y dirigido por Jorge Caldera Serrano.

Fase 2. Selección de la muestra

La muestra seleccionada para el análisis de campo ha sido:

- Periódicos digitales nacionales: 
1. Alerta Digital

2. El Confidencial

3. El Diario

4. El Huffington post

5. Libertad Digital

6. La información

7. Periodista Digital

8. Público

El motivo de la elección de estos medios digitales se debe a que son los de mayor visibilidad dentro de los que editan su cabecera a nivel nacional en la Red. Hemos tomado como referencia la página web "www.prensaescrita.com" que recopila todos los medios publicados en España.

\section{Fase 3. Trabajo de campo}

A lo largo de cinco días sucesivos de la misma semana se han analizado, en el mismo horario, los ocho periódicos digitales. Las horas de análisis y el orden han sido los siguientes:

- 16:30 Alerta Digital

- 17:00 El Confidencial

- $\quad$ 17:30 El Diario

- 18:00 El Huffington post

- 18:30 Libertad Digital

- 19:00 La Información

- 19:30 Periodista Digital

- 20:00 Público

Había que establecer un horario para determinar la nueva procedencia de la información audiovisual que se produce en un periodo de 24 horas.

La fecha de realización del análisis ha sido la semana del 2 al 6 de diciembre de 2013.

Para analizar la información audiovisual se ha elaborado una ficha, la cual incluía una serie de aspectos a tener en cuenta:

- Fecha de la primera aparición de la pieza de información audiovisual: hay muchos vídeos de noticias audiovisuales que perduran varios días publicados en el medio, sin embargo, sólo nos hemos fijado en la primera vez que estos aparecieron, anotando su título y su fecha.

- Distinción entre las fuentes internas y externas de la información audiovisual: hemos tomado como fuentes internas de los vídeos a aquellos que son de producción propia del periódico digital, mientras que las externas serían los que proceden de agencias y canales externos al medio.

- Clasificación de las fuentes externas de la información audiovisual: hemos concretado a qué empresa o canal pertenecen los elementos audiovisuales de fuentes externas. 


\section{Fase 4. Análisis de datos}

La recopilación de los datos obtenidos servirá para su posterior análisis y valoración del estudio y sus objetivos..

\section{RESULTADOS}

Una vez desarrollado el trabajo de campo se distinguen los resultados entre fuentes internas y externas, y fuentes de las cuáles se desconoce su procedencia por falta de información que nos ayude a determinar la cuestión analizada. Aunque, en un primer momento y con el fin de llegar a conclusiones sobre el primer objetivo marcado, determinamos si existe la existencia de material audiovisual en medios impresos y en línea, lo que se traduce en una hibridación de medios, tal y como se señala con posterioridad.

Se describen, brevemente, los resultados obtenidos para cada medio de comunicación digital.

Alerta Digital. Difunde información audiovisual. Cuenta tanto con fuentes propias como externas. Como fuente interna facilita videcomentarios del programa "La Ratonera" y como fuentes externas, en el periodo analizado, tan sólo utiliza el canal de YouTube SportYou.

El Confidencial. Difunde información audiovisual. Cuenta con información de la cual se desconoce la procedencia, aunque podría estimarse por tipo de montaje, duración y temática que es material procedente de agencia de noticias. Como imágenes procedentes de la propia empresa, cuenta con entrevistas en la sección "apueste x 1". Son más prolíficos en la utilización de fuentes externas, encontrando vídeos de YouTube, Vimeo, IntereconomíaTube, Vice.com, National Geographic (Canal YouTube), MadridScienticFilm (Canal YouTube), LaSexta.com, rtve.es y telecinco.es

El Diario. Difunde información audiovisual. De fuentes internas por medio del Canal YouTube del propio medio, además de Vertele. Como fuentes externa se incluyen: YouTube, Amnistía Internacional España (Canal YouTube), La Moncloa (Canal YouTube), TED, Vine, Vimeo, rtv.es (Vertele), antena3.com (Vertele), cuatro.com (Vertele), telecinco.es (Vertele), LaSexta.com (Vertele) y Telemadrid (Vertele).

El Huffinton Post. Difunde información audiovisual. No cuenta con vídeos propios del propio medio, aunque sí utiliza material de agencia que pudiera haber sido adquirido por la empresa, así como de otros medios pertenecientes al mismo grupo. Como fuentes externas utiliza a YouTube, La Moncloa (Canal YouTube), aomm.tv, rtve.es y antena3.com.

Libertad Digital. Difunde información audiovisual. Cuenta con material de procedencia desconocida sobre trailer de películas de estreno. Se podría estimar que son las propias productoras $\mathrm{y}$ especialmente las distribuidoras cinematográficas las que difunden y facilitan dicho material a los medios. Como fuente propia cuentan con su propio canal en YouTube, LDTV. En el ámbito de las 
fuentes externas difunden contenidos de las siguientes plataformas: YouTube, La Moncloa (canal YouTube), La voz del Interior (canal de Youtube), Daily Motion, NBA TV, elsotanoperdido.com y British Fashion Awards.

La Información. Difunde información audiovisual. Cuenta con fuentes propias procedente de la grabación de algunas entrevistas, y como fuentes externas difunden contenidos de YouTube, Punto Pelota (canal YouTube), La Moncloa (canal YouTube), EFE y Nature Communications.

Periodista Digital. Difunde información audiovisual. Cuenta con un canal del propio medio para la difusión del material audiovisual, PDTV (canal YouTube). Como fuentes externas hemos encontrado las siguientes fuentes: YouTube, Europapress (canal YouTube), RtvCyL (Canal YouTube), RealNews Español (Canal YouTube), la agencia AP (zoomin.tv), Zoominn.tv, rtve.es, antena3.com, cuatro.com, telecinco.com y lasexta.com.

Público. Difunde información audiovisual. Cuenta con material propio procedente del programa de la propia compañía La Tuerka, y como fuentes externas utilizada YouTube, la agencia Atlas, Europapress, Media Network, Vimeo, Fox, filmtrailer.com, Gamereactor y rtve.es.

\section{ANÁLISIS DE LOS RESULTADOS}

1. Los periódicos digitales emplean información audiovisual.

Cada día del análisis, los ocho periódicos digitales analizados cuentan con presencia de información audiovisual dentro de los contenidos ofrecidos.

Y no sólo ellos, también los diferentes espacios ligados a estos medios digitales emplean vídeos como material informativo. Son los casos de los portales 'SportYou', de Alerta Digital; 'Vanitatis', de El Confidencial; 'Vertele', de El Diario y 'Te Interesa', de La Información.

2. Los periódicos digitales utilizan fuentes de información audiovisual propias y externas.

Los periódicos digitales que se han analizado en este estudio tienen vídeos elaborados por ellos mismos y vídeos de procedencia externa al medio, adquiridos de diversas agencias o plataformas de difusión.

Aunque también tenemos que destacar que se desconoce la fuente de algunos de ellos por no ser citada por el medio, ni tener ningún indicativo en la imagen que nos permitiera averiguar de cuál se trataba. Esto nos ha ocurrido con alguno de los materiales audiovisuales de El Confidencial y con los tráiler de los estrenos de la semana del periódico Libertad Digital.

Por otra parte, dudamos también de la procedencia de algunos de los contenidos señalados como propios por el medio, en los que a pesar de contar con el logotipo del periódico en la pantalla de emisión, pensamos que podrían tratarse de imágenes tomadas de agencias de noticias como Efe o Routers, o de otros portales de vídeos. Estas sospechas están fundadas en el hecho de que, en algunos casos, la noticia 
viene firmada por el redactor y la agencia, y las fotografías que acompañan al texto también tienen la autoría de la agencia de noticias. Otro motivo de sospecha es en el caso de algunas informaciones internacionales, en las que dudamos que tengan un corresponsal presenciando y filmando el hecho en ese lugar. El Huffington Post ha sido el periódico con más cantidad de vídeos dudosos como propios, junto con Libertad Digital, que el viernes 6 de diciembre tenía con su "mosca" dos vídeos idénticos a los que en otros medios procedían de YouTube.

\section{Los periódicos digitales emplean una gran variedad de fuentes externas}

El material audiovisual de los diarios digitales procede de múltiples fuentes externas al medio, entre los que encontramos tanto agencias de noticias como portales web o vídeos de cadenas televisivas.

De los ocho periódicos analizados, la única fuente en común entre todos ellos es YouTube.

YouTube no sólo es la única fuente externa en común entre los diarios digitales analizados, sino que además, a través de este portal de vídeos difunden sus contenidos otras fuentes como es el caso de Europa Press o de La Moncloa; o el material audiovisual propio de la mitad de los periódicos online analizados, que disponen de su propio canal para difundir los vídeos elaborados por ellos mismos. Este último hecho se produce en los medios digitales El Confidencial, El Diario, Libertad Digital y Periodista Digital.

4. Existen medios digitales que realizan programas audiovisuales frecuentes. La mitad de los medios online analizados publican con cierta periodicidad, bien diaria o bien semanal, una serie de vídeos con sus programas propios. Es el caso de los diarios digitales Alerta Digital, Libertad Digital, Periodista Digital y Público, que tienen un espacio dedicado dentro de su espacio web para su programación audiovisual.

\section{CONCLUSIONES}

Los periódicos digitales que han nacido en plena Sociedad de la Información en respuesta a la demanda de medios híbridos que surge con Internet, han sabido aprovechar los recursos del medio en el que publican, y en concreto, emplear información audiovisual diaria en sus noticias.

La prensa digital tiene a su disposición una múltiple variedad de fuentes audiovisuales propias y externas al medio que les permite incorporar vídeos de distinta procedencia a sus contenidos informativos y hacerlos más atractivos. Esta explosión de fuentes de información audiovisuales que se han desarrollado gracias al entramado de la gran Red de Redes, es clave en la distinción entre unos medios y otros. La elección de los periódicos digitales entre la publicación de vídeos de la misma o de distinta procedencia, determinará su diferenciación con la competencia, haciendo que se repitan o no los mismos contenidos audiovisuales. 
En cuanto a las fuentes de información audiovisuales externas a los diarios online, cabe destacar la fuerte presencia de YouTube, portal de cuyos contenidos se nutren todos los periódicos digitales para ofrecer vídeos que acompañen y/o complementen sus noticias. Esta plataforma virtual de vídeos no sólo es una fuente externa de la prensa digital, sino que además se ha convertido en un canal de emisión y difusión del material audiovisual interno de los periódicos y de muchas agencias y compañías que distribuyen vídeos a los medios de comunicación.

Otras fuentes externas importantes dentro de la información audiovisual que ofrecen los periódicos digitales son las plataformas web de las televisiones generalistas. Los diarios publican diariamente vídeos de algunos contenidos televisivos que proporcionan estos portales de emisión de las principales cadenas de televisión, como los de Rtve, Antena 3, Cuatro, Telecinco o La Sexta.

En el caso de la información audiovisual interna, existen periódicos digitales que producen sus propios programas audiovisuales. Una parte de esta programación audiovisual online está formada por contenidos de emisoras de radio o canales de televisión pertenecientes al mismo grupo de comunicación del diario. De esta forma, aprovechan los recursos de los que disponen y, de paso, realizan una promoción mutua entre todos ellos, como ocurre con Libertad Digital y la emisora Es Radio.

A la hora de citar la procedencia de la información audiovisual que ofrece la prensa digital, existen diferencias significativas entre los distintos diarios digitales. Hay algunos que procuran especificar siempre la fuente de la que provienen los vídeos que publican, sobre todo cuando no haya nada característico que permita identificarlos. Es el caso, por ejemplo, del diario Público. Por el contrario, otros medios tienen muy descuidado este aspecto, e incluyen material audiovisual cuya procedencia es difícil, e incluso imposible, detectar.

La información audiovisual está plenamente integrada en el panorama informativo de los medios de comunicación digitales. La gran variedad de fuentes de información audiovisuales externas y de recursos virtuales disponibles para potenciar el material audiovisual propio, posibilita la distinción entre unos periódicos digitales de los otros sin tener que llegar a convertirse en ecos de la prensa tradicional.

\section{RECONOCIMIENTOS}

Este trabajo ha sido financiado por la Junta de Extremadura (Consejería de Educación, Ciencia y Tecnología) y el Fondo Social Europeo dentro del plan de apoyo a las actuaciones de los Grupos de Investigación inscritos en el catálogo de la Junta de Extremadura. GR10019. 


\section{REFERENCIAS BIBLIOGRÁFICAS}

ÁlVAREZ, Olga. Periodismo digital, periodistas digitales y empresas multimedia. Hipertext.net, 2003, $\mathrm{n}^{\mathrm{o}}$. 2, pp. 1-7.

MARTÍNEZ ALBERTOS, José Luís. El mensaje periodístico en la prensa digital. Estudios sobre el mensaje periodístico, 2001, $\mathrm{n}^{\circ} .7$, pp. 19-32.

PRENSKY, Marc. Digital Natives, Digital Immigrant. On the Horizon, 2001, vol. 9, n. 5, pp. 1-6

ROA AMAYA, Matilde. Análisis de la procedencia de las fuentes de información audiovisual en los periódicos digitales: El País, El Mundo y Hoy. Badajoz: Facultad de Ciencias de la Documentación y la Comunicación, 2011. Trabajo Fin de Máster.

ROA AMAYA, Matilde; CALDERA-SERRANO, Jorge. Uso de fuentes de información audiovisuales en los medios digitales españoles: repositorios 2.0 de vídeos. Cuadernos de Documentación Multimedia, 2012, vol. 23, pp. 128-139

SALAVERRÍA, Ramón; GARCÍA-AVILÉS, José Alberto (2008). La convergencia tecnológica en los medios de comunicación: retos para el periodismo. Trípodos, 2008, n. 23, pp. 31-47 